\title{
Proposal for an Implementation Framework for Digital Health Technology in the Intensive Care Unit: A Qualitative Study
}

\author{
Lina Mosch* \\ Charité Universitätsmedizin Berlin \\ Akira-Sebastian Poncette* \\ Charité Universitätsmedizin Berlin \\ Claudia Spies \\ Charité Universitädsmedizin Berlin \\ Steffen Weber-Carstens \\ Charité Universitätsmedizin Berlin \\ Monique Schieler \\ Charité Universitätsmedizin Berlin \\ Henning Krampe \\ Charité - Universitätsmedizin Berlin \\ Felix Balzer ( $\square$ felix.balzer@charite.de) \\ Charite Universitatsmedizin Berlin https://orcid.org/0000-0003-1575-2056
}

\section{Research}

Keywords: digital health, patient monitoring, intensive care medicine, intensive care unit, technological innovation, user-centered, usability, implementation, implementation science, qualitative research, interview

Posted Date: July 14th, 2020

DOI: https://doi.org/10.21203/rs.3.rs-40974/v1

License: (c) (i) This work is licensed under a Creative Commons Attribution 4.0 International License. Read Full License

Version of Record: A version of this preprint was published at JMIR Formative Research on August 7th, 2020. See the published version at https://doi.org/10.2196/22866. 


\section{Abstract}

Background

Intensive care is lagging with digital transformation. It is essential to provide evidence and guidelines for integrating digital health technologies into the intensive care unit (ICU).

We evaluated the implementation of a remote patient monitoring platform and derived an implementation framework proposal for digital health technology in an ICU.

Methods

We conducted this study from May 2018 to March 2020 during the implementation of a tablet-computer based remote patient monitoring system. The system was installed in the ICU of a large German university hospital as a supplementary monitoring device. Following a hybrid qualitative approach with inductive and deductive elements, we used the Consolidated Framework for Implementation Research and the Expert Recommendations for Implementing Change to analyze the transcripts of seven semi-structured interviews with ICU clinical stakeholders and quantifiable questionnaire data. Results of the qualitative analysis, together with the findings from informal meetings, field observations, and previous explorations, provided the basis for the derivation of the proposed framework.

Results

Inductive analysis of the interview transcripts revealed an insufficient implementation process because of a lack of staff engagement and little perceived benefits from the novel solution. The ICU was not considered the most suitable for remote patient monitoring, as the staff's presence and monitoring coverage were high. We propose an implementation framework for digital technologies, including strategies to apply before and during implementation, targeting the implementation setting by involving all stakeholders, assessing the intervention's adaptability, facilitating the implementation process, and maintaining a vital feedback culture. Setting up a unit responsible for implementation, taking into account the guidance of an implementation advisor, and building on existing institutional capacities could improve the institutional context of implementation projects.

Conclusions

The ICU provides an exceptional setting for the introduction of digital health technology because it is a high-tech environment involving multiple professions and high-stress levels. Before implementation, the need for innovation and the ICU's readiness to change should be assessed. During implementation, a clinical team should ensure transparent communication and continuous feedback. The establishment of an implementation unit is recommended to promote a sustainable implementation culture and to benefit from existing networks.

Trial registration

ClinicalTrials.gov NCT03514173; https://clinicaltrials.gov/ct2/show/NCT03514173

\section{Contributions To The Literature}

- We demonstrate the derivation of an implementation framework of digital health technology in the intensive care unit (ICU) by applying the Consolidated Framework for Implementation Research (CFIR) and the Expert Recommendations for Implementing Change (ERIC).

- Employing the CFIR and ERIC to analyze an ICU implementation scenario, we describe the use of these concepts in a specific implementation context, complementing relatively scarce literature on implementation science and practice in intensive care medicine.

- The proposed framework may guide health providers with concrete, evidence-based, and step-by-step recommendations for implementation practice facilitating the introduction of digital health in intensive care.

\section{Introduction}

In intensive care medicine, digital health technologies such as remote monitoring and clinical decision support systems (CDSS) promise to improve outcomes by reducing the patients' length of stay or preventing complications [1-3]. Despite its vast potential, the digital transformation of health care is lagging in numerous countries for reasons that can be ascribed at every level of the health care system. At the macro-level, weak internet infrastructures, high market fragmentation, and lack of legal frameworks, financing models and interoperability play a big role [4-6]. At the meso- and micro- levels, cumbersome operation, high costs, lack of interoperability, information governance uncertainty, and organizational resistance block digital health technologies implementation [7-10].

Numerous institutions and researchers have published guidelines and recommendations for the implementation of digital health into the health sector at the macro-level [6, 11-14]. Still scarce are the evidence regarding meso- and micro-level implementation and the guidelines for the successful integration of digital health technologies into specific clinical settings [13,15-17]. However, successful and sustainable implementation in health care requires to follow a holistic concept, applying meaningful strategies at all levels [18-20]. In particular, little research has explored the implementation processes of digital health tools in intensive care medicine, apart from the concept tele-intensive care unit (ICU) [1, 21, 22].

Five domains are essential for the implementation of digital health in various health care settings: the individual digital health technology, the outer setting, the inner setting, the individual health professionals, and the implementation process [8]. These domains were first outlined in the Consolidated Framework for

Page 2/18 
Implementation Research (CFIR), a well-proven tool to evaluate the implementation of an intervention into health care settings [9, 10, 23-26]. Targeting the improvement of implementation performance, the Expert Recommendations for Implementing Change (ERIC) provide a comprehensive compilation of strategies to boost implementation in clinical practice $[27,28]$. The CFIR domains and ERIC strategies are coherent, synergistic and provide meaningful guidance for implementation researchers and practitioners, but they require more use cases and documentation of applications in a specific context and setting. Also, the present literature on implementation strategies is extensive, unstructured, and the strategies reported are often poorly conceived [17, 29, 30].

It is not clear if it is possible to transfer the previously identified barriers and facilitators to digital health implementation into the ICU context. Clear implementation strategies for intensive care settings are still lacking.

Aim

We aimed to investigate barriers and beneficial strategies to the implementation of a remote patient monitoring technology to develop a proposal for an implementation framework for digital health technology in the ICU.

\section{Methods}

Context and Technical Setup

We conducted this qualitative study with ICU staff from a German university hospital over the course of the implementation of the Virtual Patient Monitoring Platform Vital Sync 2.4 developed by Medtronic plc, Minneapolis, United States. The device remotely monitored ICU patients from tablet computers at the hospital premises and was supplemental to the primary patient monitoring system, the IntelliVue patient monitoring system (MX800 software version M.00.03; MMS X2 software version H.15.41-M.00.04) developed by Koninklijke Philips N.V., Amsterdam, Netherlands. The primary Philips IntelliVue monitoring system displayed the vital parameters on stationary touchscreen displays at the bedside and on a monitor at the central nurse station.

The remote monitoring system was installed between May 2018 and June 2019 in five of the ten beds of the Post Anesthesia Care Unit (PACU), an ICU mainly for postoperative patients that need a short-term intensive care treatment and monitoring. The system included two sensors (the pulse oximetry and the capnography), which registered peripheral capillary oxygen saturation $\left(\mathrm{SpO}_{2}\right)$, pulse rate (PR), end-tidal carbon dioxide level (etCO $)_{2}$, and respiratory rate $(\mathrm{RR})$ at a frequency of $1 \mathrm{~Hz}$. The vital parameters were displayed on a monitor at the central nurse station and were retrievable from six tablet computers (2 large iPads, 2 iPad minis, 2 Microsoft Surfaces). A six-digit code protected the iPad access, and the data were accessible after logging into the Vital Sync website. A username and a password protected the access to the Microsoft Surfaces. Technical instructions of ICU staff (i.e., physicians, nurses and respiratory therapists) into the device were conducted over a period of one month. Additionally, two workshops were conducted for hands-on training. Additional assistance was provided as needed. Further technical description and usage of the software can be found elsewhere [31, 32].

Study Design and Research Team

We have consulted the Standards for Reporting Qualitative Research to report this research [33]. This qualitative exploratory implementation study is based on an abductive research approach as described by Dubois et al. [34,35]. The abductive approach of systematic combining (containing inductive and deductive analysis methods) specifies existing theories, refining them according to the individual case and context. We considered this approach essential to derive practical recommendations for the implementation of new technology in the ICU. The transcripts of seven semi-structured interviews and online questionnaires with key stakeholders in the clinical implementation process, the results of field observations and informal discussions among the research group, as well as findings from previous explorations were analyzed and applied to the CFIR domains and ERIC strategies to develop the proposed implementation framework (see Fig. 1). This enabled us to assess derived insights but also maintain receptivity to new aspects of the implementation process.

The research team consisted of: an MD candidate with a strong affinity for digital health (LM); a postdoctoral researcher with a background in anesthesiology, intensive care medicine, digital health and geriatrics (ASP); a professor for digital health, who is a consultant anesthesiologist and a computer scientist (FB); a psychologist (HK); the head nurse (MS); the ICU senior consultant (SWC); and the department's head of staff (CS).

Data Collection

Our data include interview transcripts and quantifiable results of a questionnaire with key stakeholders in the ICU, informal meetings and discussions among the research group, field observations, and the results of previous explorations [31, 36].

From June 2019 to November 2019, we conducted seven semi-structured interviews with ICU staff members, including three physicians, three nurses, and one respiratory therapist. The study participants were identified as key stakeholders (e.g., head nurse, senior physician) of the ICU and had closely experienced the remote patient monitoring implementation.

The interview guideline was deduced on findings of a previous study from our research group [36] and was oriented towards the categories of the CFIR (see Appendix 1). Pilot interviews with associated intensive care physicians did not alter the questions. The interviews were performed either before or after patient care and were recorded and transcribed verbatim. A researcher who had not done the transcription reviewed the transcript.

The semi-structured interview guideline included the online questionnaires containing 47 items and a technology commitment scale [37]. We used a 5-point Likert-type scale as an ordinal response format, with the options not correct at all (1), not quite correct (2), partly correct (3), quite correct (4), and completely correct (5). The study data were collected and managed using REDCap electronic data capture tools hosted at Charité - Universitätsmedizin Berlin [38, 39]. 
Informal meetings and discussions among the research group and field observations took part from the start of the implementation in May 2018 until March 2020. Results of the field research were published by Poncette et al. in 2019 [31].

\section{Data Analysis}

For qualitative analysis, we applied a hybrid approach, combining inductive and deductive coding elements, as described by Fereday et al. [40]. First, the interview transcripts were analyzed applying an inductive coding process [41]. Second, the resulting content of the codes was summarized to obtain the main findings and to serve as the basis for the deductive analysis, as described by Crabtree and Miller [42].

For deductive analysis, we used as code system templates the CFIR domains and the ERIC strategies, which are grouped into nine clusters [27, 28]. Specifically, we used the CFIR template to analyze the summaries regarding the implementation performance and the findings of the descriptive data analysis, while the ERIC strategies served as a template for analyzing the summaries of staff's suggestions on implementation process improvements. All coding was performed using MaxQDA 2020 qualitative data analysis software [43].

In the final step, the proposal for an implementing framework for digital health technology in the ICU was derived from the results of the CFIR- and ERIC-guided analyses. The CFIR-ERIC Implementation Strategy Matching Tool supported the prioritization of the derived recommendations [44]. Findings from the informal meetings, discussions, and the field observations supported in situating the results and the interview suggestions in the broader context of implementation and in supplementing objective characteristics.

Descriptive data analysis of questionnaire items was conducted using Microsoft Excel 2020 (Version 16.35).

\section{Results}

Overview

Inductive analysis of the interview transcripts revealed two major categories perceived performance of the implementation and perceived factors improving implementation, which contained four and three subtopics, respectively. According to interviewed stakeholders, the remote patient monitoring system's implementation was insufficient due to a lack of staff engagement in the process and the little perceived benefit from the novel solution in its current version. Factors suggested improving implementation were targeting staff training, features of the technology itself, and implementation setting.

Deductive coding was conducted by mapping CFIR domains and ERIC strategies onto the summaries from the inductive analysis as well as quantifiable data from the interviews. Four major CFIR domains were mapped: intervention characteristics, inner setting, individual characteristics, and process. Regarding perceived factors improving implementation, seven clusters of the ERIC framework were mapped: Use evaluative and iterative strategies, Provide interactive assistance, Adapt and tailor to context, Develop stakeholder interrelationships, Train and educate stakeholders, Support clinicians, and Change infrastructure.

To develop the proposal for an implementation framework for digital health technology in the ICU, we applied the CFIR-ERIC Implementation Strategy Matching Tool to the CFIR- and ERIC guided analysis. We ordered the findings into a temporal perspective.

Implementation Process

\section{Staff involvement and training}

The interviewees identified staff involvement and training as more targeted towards nursing staff, even though not in charge of the implementation project. Physicians were perceived to be not as engaged with the system and to have received less training. According to the interviewed stakeholders, staff members of all professional groups lacked a feeling of responsibility to continuously apply the remote patient monitoring system. Additionally, the staff was unable to identify a leading member in charge of the implementation process and longed for persisting initiatives to enhance staff engagement, such as regular staff training and information sessions. Interviewees reported that opinion leaders' communication of perceived negative features of the intervention among staff created a negative peer pressure not to use the system.

Lack of knowledge about the implementation project's aims and context and a lack of continuous staff training led to negative feelings towards the intervention. Interviewees said to have felt well informed about the project initially, but the information flow decreased equally. Training did not reach all staff members due to a complex shift system and a big pool of staff for two ICUs, whereas the system was implemented only at five bedsides out of ten on one ICU. Furthermore, the staff perceived the system as an imposition from outside the ICU and felt not to have any influence on the implementation.

\section{Additional benefit}

According to the interviewees, the staff did not perceive the system's added value as high for four reasons. First, the ICU already had a monitoring system offering remote functions (such as displaying vital parameters of different patients on all the bedside monitors), making an additional one superfluous. Second, due to the high staff presence in the ICU, the need to remotely monitor patients would be low. Third, the high patient turnover in the PACU caused a continuous connection and disconnection of the patients to the system, resulting in an increased workload for the nurses. Fourth, due to the severe conditions of the admitted patients, the reaction to an alarm had to be immediate. Remotely monitoring patients while being on a different ward or performing a clinical intervention would make the quick reaction impossible and, thus, would be without consequence.

\section{Intervention features}


Interviewees highlighted that the limited number of vital parameters monitored by the system was not sufficient to evaluate the patient's condition

satisfactorily. The system's dependency on a stable wireless network connection raised further concerns. Some interviewees perceived the tablet too large and inconvenient to use and carry in the tunic pockets. Finally, the device could get lost and would not allow patients' monitoring during their transportation because of the large module and cables.

\section{Attitude of staff}

Interviewees said to be satisfied with the current monitoring system and did not see the need for a change. ICU staff did not use the system because they lacked the habit and routine to use a remote patient monitoring technology. They were afraid of losing their break times when applying the system and of an increased staff workload (e.g., system set-up). They also feared that reduced patient contact and false alarm when applying the system might increase the stress levels and endanger patient safety. Overall, the staff saw no additional benefit in the technology.

Mapping of CFIR Domains

The summaries of the staff interview transcripts and quantifiable questionnaire responses were coded and assigned to four major domains of the CFIR: Intervention characteristics, inner setting, individual characteristics, and process (see Table 1). 
Table 1

Mapping of CFIR domains to summaries of codes concerning the implementation performances.

$\begin{array}{ll}\text { CFIR Construct } & \text { Summary segment and questionnaire responses } \\ \begin{array}{l}\text { Intervention } \\ \text { characteristics }\end{array} & \\ \begin{array}{l}\text { Intervention } \\ \text { source }\end{array} & \begin{array}{l}\text { There was a feeling among the staff that the remote patient monitoring system was being imposed from above without having an } \\ \text { influence on being part of the implementation. }\end{array}\end{array}$

source influence on being part of the implementation.

Evidence The installed beta version of the system only offered a limited number of vital parameters that could be monitored.

strength and

quality

Relative

advantage

The system was introduced to the ICU as a supplementary monitoring system, which was why the staff did not perceive its additional value as high.

The current monitoring system already offered remote monitoring functions such as flexibly, displaying parameters of different patients on a bedside monitor

In an ICU, the reaction to an alarm has to be immediate due to the severe conditions of the admitted patients.

Remotely monitoring patients while being on a different ward or in a different part of the hospital makes the quick reaction impossible and, thus, is without consequence.

The available vital signs were not sufficient to evaluate the patient's condition satisfactorily.

The majority stated that using the remote patient monitoring as a supplementary monitoring in the ICU was not useful (5 not correct at all or not quite correct), did not have a benefit for the respective professional group ( 5 not correct at all or not quite correct,) [...].

Trialability The system was introduced to the ICU as a supplementary monitoring system, which was why the staff did not perceive its additional value as high.

Adaptability The system was introduced to the ICU as a supplementary monitoring system, which was why the staff did not perceive its additional value as high.

In an ICU, the reaction to an alarm has to be immediate due to the severe conditions of the admitted patients.

Remotely monitoring patients while being on a different ward or in a different part of the hospital makes the quick reaction impossible and, thus, is without consequence.

If physicians are registering a remote alarm while being occupied with tasks such as placing a central venous catheter or performing surgery, those alarms remain without consequence.

In key situations such as transport of patients, using the system for monitoring was not possible due to a large module and several cables.

The majority stated that [...] the remote patient monitoring as a supplementary monitoring in the ICU [...] was not well integrated into the clinical routine (5 not correct at all or not quite correct).

Complexity The high patient turnover in a post-anesthesia care unit led to a higher workload for nursing staff while connecting and disconnecting the system for every new patient.

The tablet was perceived by some interviewees as too large for using it in the daily work routine.

A reason for not using the system was the inconvenience of carrying another device in already packed tunic pockets.

A concern was that the device can get lost.

Interviewees raised concerns about the system's dependency on a stable WiFi connection.

\section{Inner setting}

Structural

characteristics

Training did not reach all staff due to a complex shift system and a big pool of staff for two ICUs.

The system was implemented only at five out of ten bedsides on one out of two ICUs.

The high number of monitors in an ICU made an additional tablet not necessary, according to the interviewees.

The perceived impact of the system was low because high staff presence in the ICU made the implementation of remote patient monitoring superfluous.

The high patient turnover in a post-anesthesia care unit led to a higher workload for nursing staff while connecting and disconnecting the system for every new patient.

Respondents claimed that in an ICU, the reaction to an alarm has to be immediate due to the severe conditions of the admitted patients. Remotely monitoring patients while being on a different ward or in a different part of the hospital makes the quick reaction impossible and, thus, is without consequence.

There was a lack of persisting initiatives to engage staff in the implementation process such as regular staff training and information events.

Communication of negative aspects of the intervention created a negative peer pressure to not use the system.

Training did not reach all staff due to a complex shift system and a big pool of staff for two ICUs. 
The team spirit on the ICU was stated to be good by the majority (7 quite correct).

The general atmosphere within the ICU team had a large impact on the implementation process according to the interviewees $(5$ quite correct).

The majority stated that the aims and purpose of the remote patient monitoring implementation was clearly communicated (6 quite correct or completely correct ).

Implementation The system was introduced to the ICU as a supplementary monitoring system, which was why the staff did not perceive its climate additional value as high.

- Tension for The current monitoring system already offered remote monitoring functions, such as flexibly displaying parameters of different change patients on a bedside monitor

ICU staff saw no additional benefit in using the system.

Interviewees said to be satisfied with the current patient monitoring and do not see the need for change.

Implementation Staff involvement was perceived to be more targeted towards nursing staff, although not being in charge of the implementation climate project.

- Compatibility

Implementation climate

There was a lack of feeling of responsibility by staff members of all professions to continuously apply the system.

- Relative priority

Implementation climate

On the one hand, a leading nurse or physician was not identified for the implementation process by the staff, on the other hand, interviewees reported a lack of persisting initiatives to engage staff in the implementation process such as regular training and information events.

- Learning

climate

The high patient turnover in a post-anesthesia care unit led to a higher workload for nursing staff while connecting and disconnecting the system for every new patient.

Implementation

readiness

A lack of leadership engagement was reported both in the nursing and the medical department.

- Leadership engagement

Implementation readiness

Staff could not identify a leading nurse or physician responsible for the implementation process.

- access to

information and

knowledge

\section{Individual}

characteristics

Knowledge and beliefs about the intervention

Self-efficacy

Individual stage of change
Communication of negative aspects of the intervention created a negative peer pressure to not use the system.

A lack of knowledge about the aims and context of the implementation project, and a lack of continuous staff training led to a negative feeling towards the intervention and a lack of motivation to engage further with the system.

The staff was afraid of losing their break times when applying the remote patient monitoring system.

Another fear was that the system creates an increased workload for staff (e.g., set-up, connection) rather than decreasing it.

ICU staff claimed that remote monitoring would lead to less direct patient contact and thus threaten patient safety as the patient's clinical condition would not be evaluated adequately.

The staff feared that additional (false) alarms would appear when applying the system, increasing the stress level and endangering patient safety.

Interviewees accomplished, on average, 47 points on a 12-60 point technology commitment scale, which shows high technology commitment.

ICU staff did not use the system because they lacked the habit and routine to use remote patient monitoring technology.

ICU staff saw no additional benefit in using the system.

Opinions were split if patient monitoring improvements are necessary (3 not correct at all or not quite correct, 4 quite correct or completely correct ), if remote patient monitoring of patients in the ICU has advantages (1 not quite correct, 3 quite correct or completely correct ), and if remote patient monitoring in the ICU is important (2 not quite correct, 1 completely correct).

The majority of participants stated to be satisfied with the current patient monitoring (6 quite correct or completely correct).

\section{Process}

Planning

Engaging
There was a lack of knowledge among staff about the aims and context of the implementation project.

Staff involvement was perceived to be more targeted towards nursing staff, although not being in charge of the implementation project. 


\begin{tabular}{|c|c|}
\hline CFIR Construct & Summary segment and questionnaire responses \\
\hline & $\begin{array}{l}\text { There was a lack of persisting initiatives to engage staff in the implementation process such as regular staff training and } \\
\text { information events. }\end{array}$ \\
\hline $\begin{array}{l}\text { Engaging } \\
\text { - Opinion leaders } \\
\text { - Peers }\end{array}$ & Communication of negative aspects of the intervention created a negative peer pressure to not use the system. \\
\hline $\begin{array}{l}\text { Engaging } \\
\text { - Opinion leaders } \\
\text { - Experts }\end{array}$ & Physicians were perceived to be not as engaged with the system and to have received less training. \\
\hline $\begin{array}{l}\text { Engaging } \\
\text { - Formally } \\
\text { appointed } \\
\text { internal } \\
\text { implementation } \\
\text { leaders }\end{array}$ & Staff could not identify a leading nurse or physician responsible for the implementation process. \\
\hline Executing & $\begin{array}{l}\text { The frequency of staff training was very high in the beginning but decreased over time. Staff said to have felt well informed about } \\
\text { the project initially, however, the information flow decreased equally. }\end{array}$ \\
\hline
\end{tabular}

Intervention characteristics:

- Intervention source

- Evidence strength and quality

- Relative advantage

- Adaptability

- Trialability

- Complexity

Inner setting:

- Structural characteristics

- Networks and communication

- Implementation climate

Tension for change

Compatibility

Relative priority

Learning climate

- Implementation readiness

Leadership engagement

Access to information

Individual characteristics:

- Knowledge and beliefs about the intervention

- Self-efficacy

- Individual stage of change

Process:

- Planning

- Engaging

Opinion leaders

Formally appointed implementation leaders

- Executing

Strategies to Improve Implementation 
According to the interviewed stakeholders, persistent leadership engagement and the nomination of specific responsible persons for the implementation process were essential for successful implementation, especially in a busy environment such as the ICU. Furthermore, staff training should take place continuously before or after shifts and was particularly critical in the early implementation stages. A high frequency of staff training would increase implementation success. During training, staff should be informed about the implementation project and its aims to encourage the new technology application. The quality of the instructions was considered essential to influence the staff's opinion towards the implementation positively. Feedback discussions with staff and project leaders and a well-function team with a good spirit would increase staff engagement and be beneficial for successful implementation. Finally, the communication of the project by team leaders and coordinators should be encouraging and motivating.

\section{Infrastructure}

It was reported that to equip all beds in the ward with the technology and all staff members with portable monitoring devices would increase the implementation performance. A normal or intermediate care unit (IMCU) would be more suitable for a remote patient monitoring technology because of a lower staff presence and scarcer technical facilities. Interviewees suggested that patients with a relatively weak indication for admission to the ICU could be admitted to a normal ward or IMCU and be monitored remotely from there. They added that the implementation of technology that includes ICU patients (e.g., patient monitoring devices) would be more straightforward in a ward with more extended patient stays, as short stays imply more staff work to install the system.

\section{Intervention features}

High intuitiveness would be crucial for effective implementation, stated the interviewees. A monitoring solution without cables would increase usability and perceived benefit. Opinions on the device size varied; a clear visualization needs a large screen, but interviewees favored a device that fits into the pocket of a tunic. Software interoperability with other devices (such as the respirator or the PDMS) would be essential for a successful implementation, especially regarding the import and export of patients' data and parameter visualization on one screen. It was suggested to thematically group and display parameters, for example, by organ.

Mapping of ERIC Strategies

Of the 73 ERIC strategies, 19 were mapped to the summary segments concerning staff suggestions for implementation and quantifiable questionnaire responses (see Table 2). The segments were assigned to seven of the nine clusters of the ERIC framework. 
Table 2

ERIC strategies mapped to summaries of codes concerning staff suggestions for improving implementation performance.

\section{ERIC strategy \\ Summary segment and questionnaire responses}

Use evaluative and iterative strategies

Purposely reexamine the implementation

Develop a formal

implementation blueprint

Audit and provide

feedback

\section{Provide interactive}

assistance

Facilitation

During training, sta

echnology.

A well-functioning team with good team spirit was deemed beneficial for successful implementation.

Provide clinical Persistent leadership engagement and the nomination of specific responsible persons for the implementation process was supervision

\section{Adapt and tailor to}

context

Promote adaptability

During training, staff should be informed of the implementation project and its aims in order to increase motivation to apply the new technology.

With regard to the Software of the monitoring technology, interoperability with other devices such as the respirator or the PDMS is important for a successful implementation of a remote patient monitoring system, especially regarding import and export of patient data and visualization of parameters on one screen.

High intuitiveness is crucial for effective implementation.

A large screen for clear visualization is demanded, on the other hand interviewees favored a device that fits into the pocket of a tunic, e.g. a smartphone.

The intelligent grouping and display of monitoring parameters thematically by organ systems is advocated. Visualization of alarms should be clearer. Intelligent alarm management would be beneficial. Remote patient monitoring via smartphone could work well with vibration alarms.

Availability of all standard vital sign parameters (7 quite correct or completely correct), high intuitiveness (6 quite correct or completely correct) and high additional benefit (6 quite correct or completely correct) of the intervention would facilitate implementation.

\section{Develop}

stakeholder

interrelationships

Recruit,

designate, and

train for

leadership

Persistent leadership engagement and the nomination of specific responsible persons for the implementation process was important for successful implementation, especially in a busy environment such as the ICU.

Furthermore, communication of the project by team leaders and coordinators should be encouraging and motivating.

Organize

clinician

implementation

team meetings

Model and

simulate

change

Involve

executive

boards

Feedback discussions with staff and project leaders during implementation would increase staff engagement.

Inform local opinion leaders

Staff should be informed of the implementation project and its aims in order to increase motivation to apply the new technology

Persistent leadership engagement and the nomination of specific responsible persons for the implementation process was important for successful implementation, especially in a busy environment such as the ICU.

Persistent leadership engagement and the nomination of specific responsible persons for the implementation process was important for successful implementation, especially in a busy environment such as the ICU.

Feedback discussions with staff and project leaders during implementation would increase staff engagement.

Identify and

prepare

champions

Persistent leadership engagement and the nomination of specific responsible persons for the implementation process was important for successful implementation, especially in a busy environment such as the ICU.

Furthermore, communication of the project by team leaders and coordinators should be encouraging and motivating 


\begin{tabular}{|c|c|}
\hline ERIC strategy & Summary segment and questionnaire responses \\
\hline \multicolumn{2}{|l|}{$\begin{array}{l}\text { Train and educate } \\
\text { stakeholders }\end{array}$} \\
\hline \multirow{2}{*}{$\begin{array}{l}\text { Conduct } \\
\text { ongoing } \\
\text { training }\end{array}$} & $\begin{array}{l}\text { Furthermore, staff training should take place continuously before or after shifts and was particularly important in early } \\
\text { implementation stages. }\end{array}$ \\
\hline & High frequency of staff training would increase implementation success (5 quite correct or completely correct, 1 not quite correct). \\
\hline \multirow{2}{*}{$\begin{array}{l}\text { Make training } \\
\text { dynamic }\end{array}$} & The quality of the instructions was essential to positively influence the staff's opinion towards the implementation. \\
\hline & Furthermore, communication of the project by team leaders and coordinators should be encouraging and motivating. \\
\hline $\begin{array}{l}\text { Conduct } \\
\text { educational } \\
\text { meetings }\end{array}$ & $\begin{array}{l}\text { The personnel should be informed of the implementation project and its aims in order to increase motivation to apply the new } \\
\text { technology. }\end{array}$ \\
\hline \multicolumn{2}{|l|}{ Support clinicians } \\
\hline $\begin{array}{l}\text { Remind } \\
\text { clinicians }\end{array}$ & $\begin{array}{l}\text { Furthermore, staff training should take place continuously before or after shifts and was particularly important in early } \\
\text { implementation stages. High frequency of staff training increased implementation success. During training, staff should be } \\
\text { informed of the implementation project and its aims in order to increase motivation to apply the new technology. The quality of the } \\
\text { instructions was essential to positively influence the staff's opinion towards the implementation. Feedback discussions with staff } \\
\text { and project leaders during implementation would increase staff engagement. }\end{array}$ \\
\hline $\begin{array}{l}\text { Facilitate relay } \\
\text { of clinical data } \\
\text { to providers }\end{array}$ & $\begin{array}{l}\text { During training, staff should be informed of the implementation project and its aims in order to increase motivation to apply the } \\
\text { new technology. The quality of the instructions was essential to positively influence the staff's opinion towards the implementation. } \\
\text { Feedback discussions with staff and project leaders during implementation would increase staff engagement. }\end{array}$ \\
\hline \multicolumn{2}{|l|}{$\begin{array}{l}\text { Change } \\
\text { infrastructure }\end{array}$} \\
\hline \multirow[t]{2}{*}{$\begin{array}{l}\text { Change service } \\
\text { sites }\end{array}$} & $\begin{array}{l}\text { A normal ward or IMCU would be more suitable for a remote patient monitoring technology, as staff presence is lower and technical } \\
\text { facilities are scarcer. Patients with a relatively weak indication for admission to the intensive care unit, such as postoperative } \\
\text { monitoring in a patient with sleep apnea, could thus be admitted to normal ward or IMCU. }\end{array}$ \\
\hline & $\begin{array}{l}\text { Wards with low staff turnover ( } 6 \text { quite correct or completely correct) and low patient turnover ( } 5 \text { quite correct or completely correct) } \\
\text { would be more suitable according to the majority. }\end{array}$ \\
\hline \multirow{2}{*}{$\begin{array}{l}\text { Change } \\
\text { physical } \\
\text { structure and } \\
\text { equipment }\end{array}$} & $\begin{array}{l}\text { To increase implementation performance, all beds on the ward should be equipped with the respective technology, equally all staff } \\
\text { members should have access to a portable monitoring device (tablet, smartphone). }\end{array}$ \\
\hline & $\begin{array}{l}\text { The majority stated ubiquitous availability of the technology (all beds equipped) would facilitate implementation (7 quite correct or } \\
\text { completely correct). }\end{array}$ \\
\hline
\end{tabular}

Use evaluative and iterative strategies

- Purposely re-examine the implementation

- Develop a formal implementation blueprint

- Audit and provide feedback

Provide interactive assistance

- Facilitation

- Provide clinical supervision

Adapt and tailor to context

- Promote adaptability

Develop stakeholder interrelationships

- Identify and prepare champions

- Organize clinician implementation team meetings

- Recruit, designate and train for leadership

- Inform local opinion leaders

- Model and simulate change

- Involve executive boards

Train and educate stakeholders

- Conduct ongoing training

- Make training dynamic

- Use train-the-trainer strategies 
- Conduct educational meetings

Support clinicians

- Facilitate relay of clinical data to providers

- Remind clinicians

Change infrastructure

- Change physical structure and equipment

- Change service sites

Proposal for an Implementation Framework for Digital Health Technology in the ICU

The developed implementation framework includes eleven recommendations derived from ERIC strategies belonging to four clusters of the ERIC framework. A temporal perspective was added, and recommendations were specified to the ICU environment (Fig. 2). Our recommendations are targeted towards hospital administrations, leading clinicians in the ICU, and implementation researchers, meaning persons responsible for the implementation process of new digital health technology in the ICU. Before implementing, seven strategies, such as Conduct local needs assessment, Visit other sites, or Promote adaptability, should be completed. During the implementation process, we recommend applying the ERIC strategies Facilitation and Audit and provide feedback continuously. The strategies Promote network weaving and Use an implementation advisor should optimize the implementation setting's context. Optimally, an implementation unit with experts for the local implementation characteristics should be established. Several factors influence the choice of the time to start the implementation process, and an implementation advisor should be consulted to adapt these factors to the context and local needs. Regular feedback by ICU staff regarding the implementation process can lead to further need for innovation and ideas to implement digital health technologies (feedback loop, Fig. 2).

\section{Discussion}

Principal findings

Taking the example of a remote patient monitoring system, the study confirmed critical barriers to the implementation of new digital health technologies [8, 10 , 46] and showed that these also apply to the intensive care setting. The proposed implementation framework for digital health technology in the ICU includes practical strategies to overcome these barriers while using previously identified facilitators that can be applied before, during, and in the general context of an implementation.

Before implementation and in the general context, sharing use cases and building upon existing best-practices are crucial strategies to adapt and choose the technology that best fits the local settings (i.e., Visit other sites, Promote network weaving, and Use an implementation advisor) [10, 18]. Initiators of an implementation project should lay out its details, aim, and context before implementation (Develop a formal implementation blueprint). Transferable discoveries from these strategies and the strategies we propose to be applied before implementation (Promote adaptability, Conduct local needs assessment, Assess for readiness, and identify barriers and facilitators) could be used to improve the adaptability and needs-orientation of the intervention. Adaptability and user-centered design have been identified as key facilitators of digital health implementation in other settings $[8,46,47]$. To create the respective conditions, developers and providers of digital health technologies should actively participate in the implementation processes by taking advantage of the valuable feedback from clinical stakeholders and adapting their products in the spirit of user-centered design [48-50]. Therefore, our proposed implementation framework suggests several strategies to enhance the involvement of clinical stakeholders directly (Organize clinician implementation team meetings, Inform local opinion leaders, Identify and prepare champions), in line with proposed strategies for other implementation settings [51, 52].

During implementation, ensuring a transparent communication of the project's aim and context (Audit and provide feedback) is as critical as an effective Facilitation to improve staff involvement and to promote and sustain implementation.

Sustainable implementation practice means to include the aims and the strategies mentioned above in the general context of implementation practice. We propose the strategies Use an implementation advisor and Establish an implementation unit to improve the implementation environment and the local conditions for a fast, efficient and sustainable implementation of technology that focuses on the needs of users and patients and adds value. These processes should always be re-evaluated to re-adapt interventions following the changing needs [51, 53, 54].

Implementing Technology in the ICU

For decades, the ICU has been high-tech equipped to support staff with continuous monitoring of patients' vital signs, application of medication, documentation (e.g., PDMS), or diagnostics (e.g., ultrasound, bronchoscopy). However, the implementation of innovative technology in a demanding and hectic environment, such as the ICU, is a challenge [55]. This has prominently been shown by various projects, more recently, through the rise of telemedicine in the ICU [56], necessitating frameworks for the implementation of such endeavors.

Reported digital health implementation efforts in the ICU rarely involved the use of developed implementation frameworks [57]. This could be due to a lack of both implementation expert consultation and framework transferability into clinical routine. Current frameworks for the design and implementation of digital health technologies are based on best practices and, if evidence-based, need to be validated [27, 58]. Our study provides the first approach to target implementation challenges and to optimize innovation flows and adaptability in the complex environment of an ICU. Further optimization by saturating

Page $12 / 18$ 
theories with practical experiences from clinical translation is crucial for the development of a scalable and agile framework for the implementation of digital health technology in the ICU.

Implementation Units

In the decades to come, health care providers will experience the need to implement new digital health technology into their infrastructures [56, 59-61]. They will necessitate to be abreast of the latest digital health technologies, to select the appropriate technology for the specific area of application, and to plan and execute the implementation process, requiring an effective and efficient approach to implementation.

The question arises as to which staff position is responsible for overseeing, evaluating, and adapting recent evidence and strategies in implementation science to the local context. As suggested, internal and external implementation experts should be involved as early as possible [27]. With the introduction of a unit for implementation as a central starting point for any implementation project, resources for redundant project planning or ineffective implementation could be spared and invested elsewhere. To what extent these units will be involved in the ICU design, for example, should be individually assessed. Beyond the consultation and proposal regarding innovations, such a unit could assess the usability of devices and the adaptability of the intervention before procurement [62] or foster exnovation and de-implementation of outdated or useless technology.

Limitations

Our study provides valuable insights into the process of implementing digital health technology in the ICU and highlights important application strategies while planning an implementation project. However, the research team could only gather a limited perspective on the implementation project. We could not evaluate the outer setting and the manufacturer's perspective.

A limitation to the study's scope is that the ERIC strategies do not include changes in intervention characteristics, which would be essential when aiming to improve implementation performance in user-centered design. ERIC only covers the last stages of implementation (planning and executing the implementation of the finalized intervention) but does not include the re-adaptation of the intervention as part of the development process.

The CFIR-ERIC mapping tool needs further validation and evidence. Thus, the mapping of strategies to the major barriers might not reflect the best strategy to tackle the respective barrier. We sought to overcome this limitation through profound discussions at meetings within the research team, extensive field research, and through the analysis of suggestions from staff for improving the implementation performance.

Finally, the fact that every ICU has unique structural and social features, as well as the number of interviewees, could limit the general validity of derived findings. As we investigated an explicit use case in an ICU, potential interviewees were limited since we identified and interviewed the key stakeholders throughout the study. We believe that this study depicts an implementation project that is close to the standard practice in Germany, where implementation science is still an evolving discipline.

\section{Conclusion}

We propose an implementation framework for digital technology in the ICU, which entails practical and evidence-based strategies to improve the implementation process. The ICU provides an exceptional setting for the introduction of digital health technology; it represents a high-stress level for staff, and it is composed of a multi-professional team using the same technologies. This is an indispensable feature and will become even more critical in the future.

The proposed framework outlines strategies to be applied before, during, and in the general context of implementation. Before implementation, the need for innovation and potential interventions should be carefully assessed by involving all clinical stakeholders. Interventions should be needs-oriented, usercentered, and adaptable to changing circumstances. During implementation, a clinical implementation team should ensure transparent staff communication regarding the project and continuous feedback through local opinion leaders and champions. To ensure efficient management of resources and time, we recommend optimizing the general context of implementation practice in the ICU and the health care institution by involving an implementation advisor, ideally in consultation with an implementation unit of the same institution. Our proposed framework should encourage other health care institutions to implement modern digital technology in their clinical routine in ICUs.

\section{Abbreviations}

CFIR

Consolidated Framework for Implementation Research

DFG

Deutsche Forschungsgemeinschaft

ERIC

Expert Recommendations for Implementing Change

etCO ${ }_{2}$ end-tidal carbon dioxide level

ICU

Intensive Care Unit

IMCU

Intermediate Care Unit

PACU

Page $13 / 18$ 
Post Anesthesia Care Unit

PDMS

Patient Data Management System

PR

pulse rate

RR

respiratory rate

$\mathrm{SpO}_{2}$

peripheral capillary oxygen saturation

\section{Declarations}

\section{Ethics approval and consent to participate}

The ethical approval for this study was granted by the Ethics Commission of the Charité - Universitätsmedizin Berlin (EA1/031/18). Participation in the survey was voluntary. Before the study, all participants gave their consent.

\section{Consent for publication}

Not applicable.

\section{Availability of data and materials}

The datasets generated and analyzed during this study are not publicly available due to reasons of data privacy; however, they are available from the corresponding author (FB) upon reasonable request.

\section{Competing interests}

CS and FB report funding from Medtronic. The other authors do not have competing interests to declare.

\section{Funding}

Not applicable.

Authors' contributions

CS had the idea for shared decision allocation and initiated the implementation of remote patient monitoring in the intensive care unit. The study was conceived by ASP, CS, FB and LM. LM conducted data acquisition and analysis, supported by ASP. LM and ASP wrote the manuscript. HK contributed to the study's methodology and interpretation of results from a psychologist's point of view. FB supervised all parts of the study. All authors critically reviewed and approved the manuscript.

\section{Acknowledgements}

We express our gratitude to the ICU staff for their participation in this study. We would like to thank the German Research Foundation (DFG), the Open Access Publication Funds of the Charité - Universitätsmedizin Berlin, and the Einstein Center Digital Future. We would further like to thank Medtronic for providing the aforementioned devices free of charge, and F. Cappellini for copy-editing the manuscript.

\section{References}

1. Kumar S, Merchant S, Reynolds R. Tele-ICU: Efficacy and Cost-Effectiveness of Remotely Managing Critical Care. Perspect Health Inf Manag AHIMA Am Health Inf Manag Assoc [Internet]. 2013 [cited 2019 Jan 21];10. Available from: https://www.ncbi.nlm.nih.gov/pmc/articles/PMC3692325/.

2. Hawkins HA, Lilly CM, Kaster DA, Groves RH, Khurana H. ICU Telemedicine Comanagement Methods and Length of Stay. Chest. 2016;150:314-9.

3. Meyer A, Zverinski D, Pfahringer B, Kempfert J, Kuehne T, Sündermann SH, et al. Machine learning for real-time prediction of complications in critical care: a retrospective study. Lancet Respir Med. Elsevier; 2018;6:905-14.

4. Thiel R, Deimel L, Schmidtmann D, Piesche K, Hüsing T, Rennoch J, et al. \#SmartHealthSystems - International comparison of digital strategies [Internet]. Gütersloh, Germany: Bertelsmann Stiftung; 2018. Available from: https://www.bertelsmannstiftung.de/fileadmin/files/Projekte/Der_digitale_Patient/VV_SHS-Studie_EN.pdf.

5. Bughin J, Hazan E, Labaye E, Manyika J, Dahlström P, Ramaswamy S, et al. Digital Europe: Realizing the continent's potential | McKinsey [Internet]. 2016 [cited 2019 Jan 25]. Available from: 
https://www.mckinsey.com/ /media/McKinsey/Business\%20Functions/McKinsey\%20Digital/Our\%20Insights/Digital\%20Europe\%20Pushing\%20the\%20 Europe-Full-report-June-2016.ashx.

6. European Commission. Communication on enabling the digital transformation of health and care in the Digital Single Market; empowering citizens and building a healthier society [Internet]. Digit. Single Mark. - Eur. Comm. 2018 [cited 2019 Jun 22]. Available from:

https://ec.europa.eu/newsroom/dae/document.cfm?doc_id=51628.

7. Wachter RM. Making IT, Work: Harnessing the Power of Health Information Technology to Improve Care in England [Internet]. 2016. Available from: https://www.basw.co.uk/system/files/resources/basw_70727-3_0.pdf.

8. 10.1186/s13012-016-0510-7

Ross J, Stevenson F, Lau R, Murray E. Factors that influence the implementation of e-health: a systematic review of systematic reviews (an update). Implement Sci [Internet]. 2016 [cited 2019 Jan 15];11. Available from: http://implementationscience.biomedcentral.com/articles/10.1186/s13012-0160510-7.

9. Marcial LH, Johnston DS, Shapiro MR, Jacobs SR, Blumenfeld B, Rojas Smith L. A qualitative framework-based evaluation of radiology clinical decision support initiatives: eliciting key factors to physician adoption in implementation. JAMIA Open Oxford Academic. 2019;2:187-96.

10. Lennon MR, Bouamrane M-M, Devlin AM, O'Connor S, O'Donnell C, Chetty U, et al. Readiness for Delivering Digital Health at Scale: Lessons From a Longitudinal Qualitative Evaluation of a National Digital Health Innovation Program in the United Kingdom. J Med Internet Res [Internet]. $2017 ; 19$. Available from: https://www.ncbi.nlm.nih.gov/pmc/articles/PMC5334516/.

11. World Health Organization. WHO guideline: recommendations on digital interventions for health system strengthening. 2019.

12. EXpert Panel on effective ways of investing in Health. Assessing the impact of digital transformation of health services [Internet]. Publications Office of the European Union; 2019 [cited 2019 Feb 1]. Available from:

https://ec.europa.eu/health/expert_panel/sites/expertpanel/files/docsdir/022_digitaltransformation_en.pdf.

13. World Health Organization, International Telecommunication Union. National eHealth Strategy Toolkit [Internet]. 2012 [cited 2019 May 8]. Available from: https://apps.who.int/iris/bitstream/handle/10665/75211/9789241548465_eng.pdf?sequence=1\&isAllowed=y.

14. Balas EA, Chapman WW. Road Map For Diffusion Of Innovation In Health Care. Health Aff (Millwood). 2018;37:198-204.

15. Chambers DA, Glasgow RE, Stange KC. The dynamic sustainability framework: addressing the paradox of sustainment amid ongoing change. Implement Sci. 2013;8:117.

16. Giger JT, Pope ND, Vogt HB, Gutierrez C, Newland LA, Lemke J, et al. Remote patient monitoring acceptance trends among older adults residing in a frontier state. Comput Hum Behav. 2015;44:174-82.

17. 10.3389/fpubh.2019.00003/full

Powell BJ, Fernandez ME, Williams NJ, Aarons GA, Beidas RS, Lewis CC, et al. Enhancing the Impact of Implementation Strategies in Healthcare: A Research Agenda. Front Public Health [Internet]. Frontiers; 2019 [cited 2020 May 10];7. Available from: https://www.frontiersin.org/articles/10.3389/fpubh.2019.00003/full.

18. Aanestad M, Jensen TB. Building nation-wide information infrastructures in healthcare through modular implementation strategies. J Strateg Inf Syst. 2011;20:161-76.

19. Van Dyk L. A Review of Telehealth Service Implementation Frameworks. Int J Environ Res Public Health Multidisciplinary Digital Publishing Institute. 2014;11:1279-98.

20. Eom D, Lee H. A holistic approach to exploring the divided standards landscape in E-Health research. 2017 ITU Kaleidosc Chall Data-Driven Soc ITU K [Internet]. Nanjing: IEEE; 2017 [cited 2020 May 23]. p. 1-7. Available from: http://ieeexplore.iee.org/document/8246985/.

21. Larinkari S, Liisanantti JH, Ala-Lääkkölä T, Meriläinen $M$, Kyngäs H, Ala-Kokko T. Identification of tele-ICU system requirements using a content validity assessment. Int J Med Inf. 2016;86:30-6.

22. Moeckli J, Cram P, Cunningham C, Reisinger HS. Staff acceptance of a telemedicine intensive care unit program: A qualitative study. J Crit Care. 2013;28:890-901.

23. Damschroder LJ, Aron DC, Keith RE, Kirsh SR, Alexander JA, Lowery JC. Fostering implementation of health services research findings into practice: a consolidated framework for advancing implementation science. Implement Sci. 2009;4:50.

24. $10.1186 / 1748-5908-8-51$

Damschroder LJ, Lowery JC. Evaluation of a large-scale weight management program using the consolidated framework for implementation research (CFIR). Implement Sci [Internet]. 2013 [cited 2019 Jan 19];8. Available from: http://implementationscience.biomedcentral.com/articles/10.1186/17485908-8-51.

25. 10.1186/s12913-017-2660-y

Mwanza M, Michel C, Hirschhorn L. Improving data quality across 3 sub-Saharan African countries using the Consolidated Framework for Implementation Research (CFIR): results from the African Health Initiative. BMC Health Serv Res [Internet]. 2017 [cited 2019 Jan 19];17. Available from: https://bmchealthservres.biomedcentral.com/articles/10.1186/s12913-017-2660-y.

26. Abbott PA, Foster J, Marin H, de F, Dykes. PC. Complexity and the science of implementation in health IT-Knowledge gaps and future visions. Int J Med Inf. 2014;83:e12-22.

27. Powell BJ, Waltz TJ, Chinman MJ, Damschroder LJ, Smith JL, Matthieu MM, et al. A refined compilation of implementation strategies: results from the Expert Recommendations for Implementing Change (ERIC) project. Implement Sci. 2015;10:21. 
28. Waltz TJ, Powell BJ, Matthieu MM, Damschroder LJ, Chinman MJ, Smith JL, et al. Use of concept mapping to characterize relationships among implementation strategies and assess their feasibility and importance: results from the Expert Recommendations for Implementing Change (ERIC) study. Implement Sci. 2015;10:109.

29. Damschroder L, Powell B, Waltz T. Expert Recommendations for Tailoring Strategies to Context [Internet]. 2015 [cited 2020 Apr 20]. Available from: https://www.hsrd.research.va.gov/for_researchers/cyber_seminars/archives/1060-notes.pdf.

30. Proctor EK, Powell BJ, McMillen JC. Implementation strategies: recommendations for specifying and reporting. Implement Sci. 2013;8:139.

31. 10.1007/978-3-030-22649-7_43

Poncette A-S, Meske C, Mosch L, Balzer F. How to Overcome Barriers for the Implementation of New Information Technologies in Intensive Care Medicine. In: Yamamoto S, Mori H, editors. Hum Interface Manag Inf Inf Intell Syst [Internet]. Cham: Springer International Publishing; 2019 [cited 2019 Nov 13]. p. 534-46. Available from: http://link.springer.com/10.1007/978-3-030-22649-7_43.

32. Vital Sync ${ }^{\text {TM }}$ Virtual Patient Monitoring Platform. 2.6 | Medtronic [Internet]. [cited 2019 Jan 15]. Available from: https://www.medtronic.com/covidien/enus/products/health-informatics-and-monitoring/vital-sync-virtual-patient-monitoring-platform-2-6.html.

33. O'Brien BC, Harris IB, Beckman TJ, Reed DA, Cook DA. Standards for Reporting Qualitative Research: A Synthesis of Recommendations. Acad Med. 2014;89:1245-51.

34. Dubois A, Gadde L-E. Systematic combining: an abductive approach to case research. J Bus Res. 2002;55:553-60.

35. Zainal Z. Case Study As a Research Method. J Kemanus [Internet]. 2007 [cited 2020 Apr 23];5. Available from: https://jurnalkemanusiaan.utm.my/index.php/kemanusiaan/article/view/165.

36. Poncette A-S, Spies C, Mosch L, Schieler M, Weber-Carstens S, Krampe H, et al. Clinical Requirements of Future Patient Monitoring in the Intensive Care Unit: Qualitative Study. JMIR Med Inform. 2019;7:e13064

37. Neyer FJ, Felber J, Gebhardt C. Entwicklung und Validierung einer Kurzskala zur Erfassung von Technikbereitschaft. Diagnostica. 2012;58:87-99.

38. Harris PA, Taylor R, Minor BL, Elliott V, Fernandez M, O'Neal L, et al. The REDCap consortium: Building an international community of software platform partners. J Biomed Inform. 2019;95:103208.

39. Harris PA, Taylor R, Thielke R, Payne J, Gonzalez N, Conde JG. Research electronic data capture (REDCap)-A metadata-driven methodology and workflow process for providing translational research informatics support. J Biomed Inform. 2009;42:377-81.

40. Fereday J, Muir-Cochrane E. Demonstrating Rigor Using Thematic Analysis: A Hybrid Approach of Inductive and Deductive Coding and Theme Development. Int J Qual Methods. 2006;5:80-92.

41. Boyatzis RE. Transforming Qualitative Information: Thematic Analysis and Code Development. London: SAGE; 1998.

42. Crabtree BF, Miller WL. A template approach to text analysis: Developing and using codebooks. Doing Qual Res. Thousand Oaks, CA, US: SAGE; 1999. p. $163-177$.

43. Software VERBI. MAXQDA 2020 [Internet]. Berlin, Germany; 2019. Available from: maxqda.com.

44. Strategy Design [Internet]. Consol. Framew. Implement. Res. 2016 [cited 2020 Apr 26]. Available from: https://cfirguide.org/choosing-strategies/.

45. Harrison JP. Essentials of Strategic Planning in Healthcare. Health Administration Press; 2016.

46. Aref-Adib G, McCloud T, Ross J, O'Hanlon P, Appleton V, Rowe S, et al. Factors affecting implementation of digital health interventions for people with psychosis or bipolar disorder, and their family and friends: a systematic review. Lancet Psychiatry. 2019;6:257-66.

47. Labrique AB, Wadhwani C, Williams KA, Lamptey P, Hesp C, Luk R, et al. Best practices in scaling digital health in low and middle income countries. Glob Health. 2018;14:103.

48. Poncette A-S, Mosch L, Spies C, Schmieding M, Schiefenhövel F, Krampe H, et al. Improvements in Patient Monitoring for the Intensive Care Unit: Survey Study. J Med Internet Res. 2020.

49. Chochoiek N. Explaining the Success of User-Centered Design - An Empirical Study across German B2C Firms. Jr Manag Sci. 2017;Bd. 2 Nr. 1 (2017)-.

50. Andrade E, Quinlan LR, Harte R, Byrne D, Fallon E, Kelly M, et al. Investigation of the Human Factors, Usability and User Experience of Patient Monitors used in a Hospital Setting. Springer; 2018. p. 352-7.

51. Ross J, Stevenson F, Dack C, Pal K, May C, Michie S, et al. Developing an implementation strategy for a digital health intervention: an example in routine healthcare. BMC Health Serv Res. 2018;18:794.

52. Leeman J, Birken SA, Powell BJ, Rohweder C, Shea CM. Beyond "implementation strategies": classifying the full range of strategies used in implementation science and practice. Implement Sci. 2017;12:125.

53. Shaw J, Agarwal P, Desveaux L, Palma DC, Stamenova V, Jamieson T, et al. Beyond "implementation": digital health innovation and service design. Npj Digit Med Nature Publishing Group. 2018;1:1-5.

54. Halpern NA. Innovative designs for the smart ICU: part 1: from initial thoughts to occupancy. Chest. 2014;145:399-403.

55. Broens S, Dahan A, van Velzen M. Challenges and Pitfalls With a Randomized Clinical Trial in the Postanesthesia Care Unit [Internet]. 1 Oliver's Yard, 55 City Road, London EC1Y 1SP United Kingdom: SAGE Publications Ltd; 2020 [cited 2020 Apr 16]. Available from:

https://methods.sagepub.com/case/challenges-pitfalls-randomized-clinical-trial-postanesthesia-care-unit.

56. Koenig MA, editor. Telemedicine in the ICU [Internet]. Cham: Springer International Publishing; 2019 [cited 2019 Jun 23]. Available from: http://link.springer.com/10.1007/978-3-030-11569-2.

57. Moullin JC, Sabater-Hernández D, Fernandez-Llimos F, Benrimoj SI. A systematic review of implementation frameworks of innovations in healthcare and resulting generic implementation framework. Health Res Policy Syst. 2015;13:16.

Page 16/18 
58. Ray JM, Ratwani RM, Sinsky CA, Frankel RM, Friedberg MW, Powsner SM, et al. Six habits of highly successful health information technology: powerful strategies for design and implementation. J Am Med Inform Assoc. 2019;26:1109-14.

59. Topol E. Preparing the healthcare workforce to deliver the digital future [Internet]. 2019. Available from: https://topol.hee.nhs.uk/wp-content/uploads/HEETopol-Review-2019.pdf.

60. Luetz A, Grunow JJ, Mörgeli R, Rosenthal M, Weber-Carstens S, Weiss B, et al. Innovative ICU Solutions to Prevent and Reduce Delirium and PostIntensive Care Unit Syndrome. Semin Respir Crit Care Med. Thieme Medical Publishers; 2019;40:673-86.

61. Ince C. Physiology and technology for the ICU in vivo. Crit Care. 2019;23:126.

62. Human Factors Engineering Lab - Overview [Internet]. Mayo Clin. [cited 2020 May 9]. Available from: https://www.mayo.edu/research/labs/humanfactors-engineering/overview.

\section{Figures}

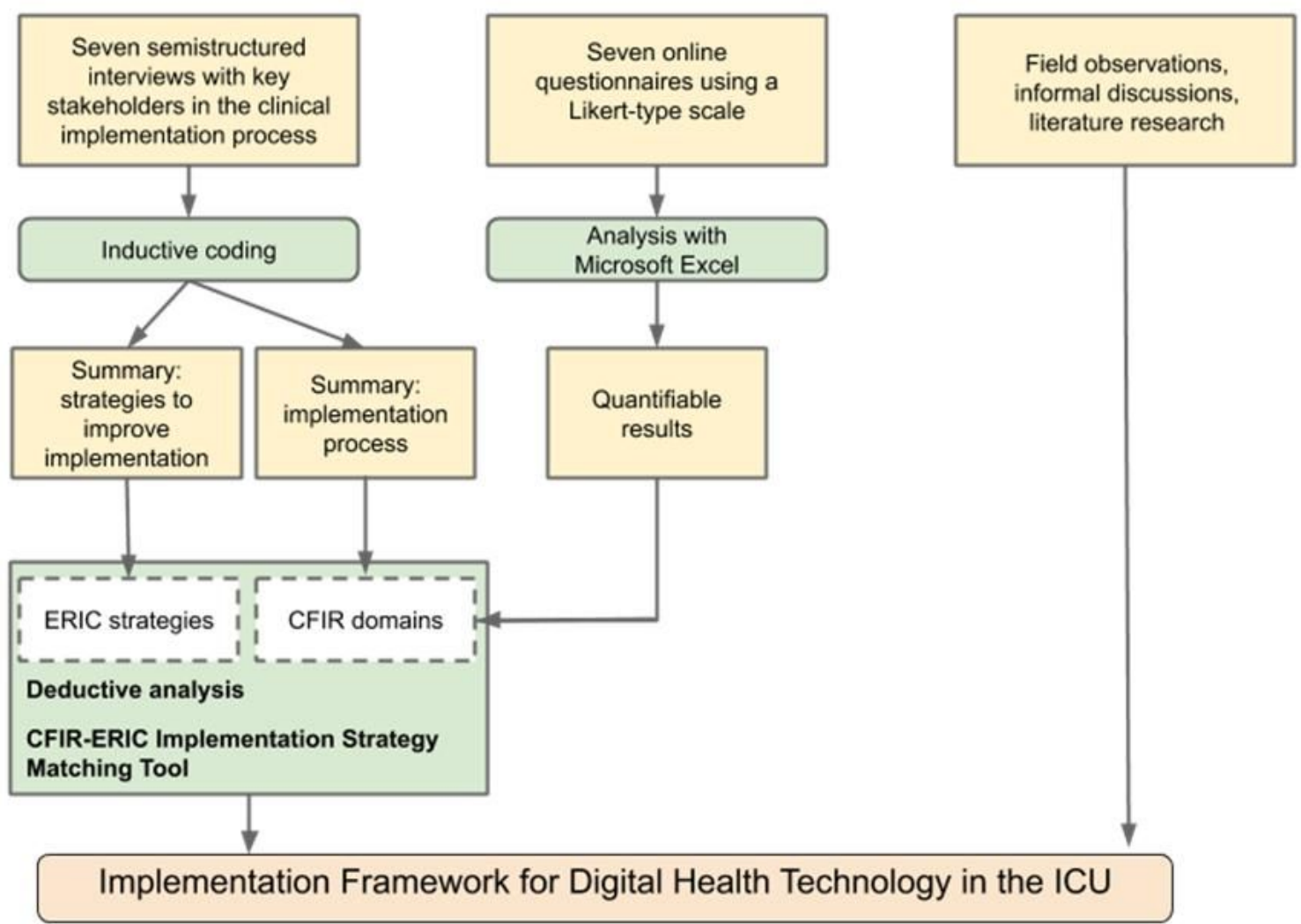

\section{Figure 1}

Overview of the data collection and analysis for the derivation of the proposed implementation framework for digital health technology in the ICU. 


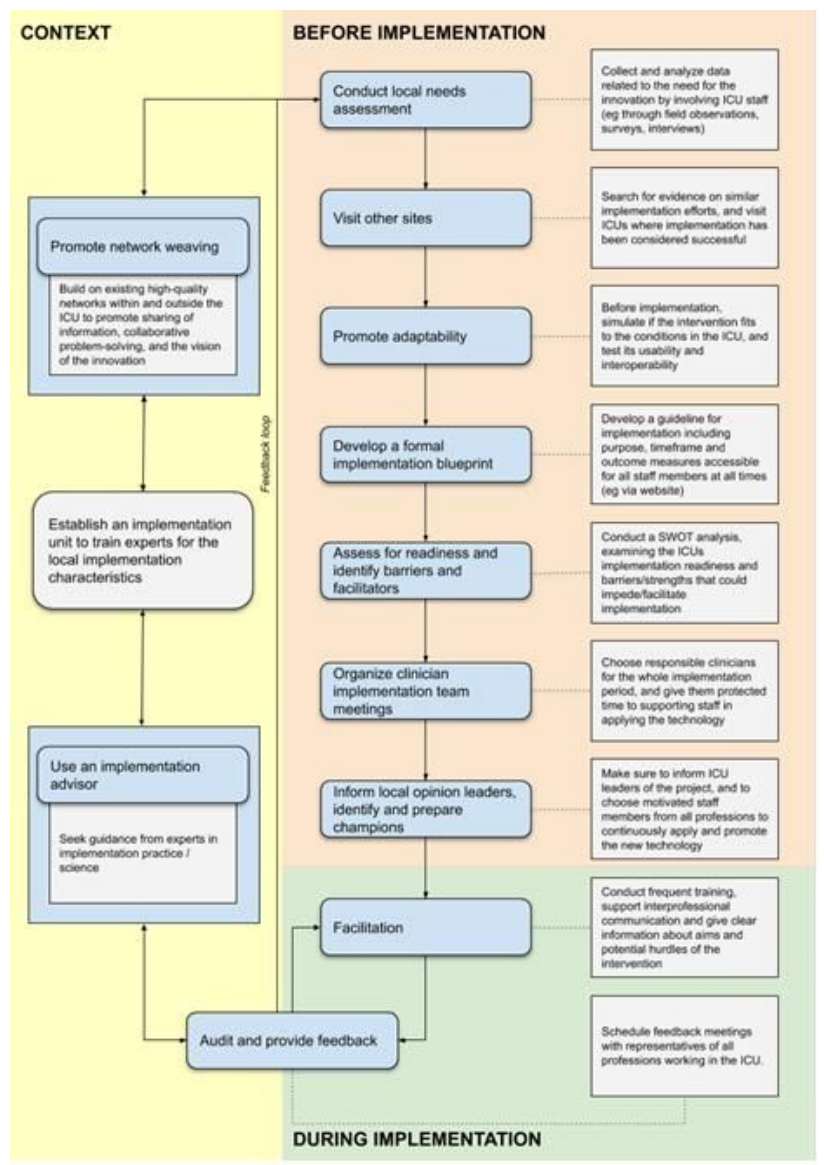

Figure 2

Strategies resulting from the CFIR-ERIC Implementation Strategy Matching Tool, and the mapping of staff suggestions for improving implementation to the ERIC strategies before (orange), during (green) implementation, and in the general context of the implementation (yellow). SWOT=Strengths Weaknesses Opportunities Threats [45]

\section{Supplementary Files}

This is a list of supplementary files associated with this preprint. Click to download.

- renamedf731c.pdf

- SRQRChecklist2.pdf

- 200606COISpiesBMC

- supplement4

- coidisclosurebalzer20200703sarpi2.pdf

- InterviewGuidelineENGfinal.docx 